\title{
Spatial and evolutionary aspects of nucleation in diffusing-reacting systems
}

\author{
M. Prieto, L. Fernández-Diaz and S. López-Andrés \\ Dpto. Cristalografia y Mineralogía, Universidad Complutense, 28040 Madrid, Spain
}

Nucleation behaviour in diffusing-reacting systems involves spatial phenomena that äre explainable by means of spatial considerations. Particularly, precipitate locations may be justified from the profiles of different physico-chemical parameters at the nucleation time. However, this instantaneous observation is not enough to explain other aspects (nucleation density, metastability level, etc.) that are in relation with the system evolution. Evolutionary variables must be introduced to account for experiments in systems of this kind. In this paper a parameter, the supersaturation rate, is quantified from experimental data of supersaturation evolution. The study is applied to growth of barium and strontium carbonates in a U-tube gel system. Supersaturation rates have an important bearing on the supersaturation level at the nucleation time and consequently on the nucleation density.

\section{Introduction}

Gel growth systems usually are finite diffusing-reacting systems in which the growth conditions continuously change in space and time. Working with these growth media, the experiments must be described by attending to the starting boundary conditions and to the system history, that is to say, the succession in time of the physico-chemical conditions everywhere in the system. The growth process must also be referred to in historical terms, by considering the sequence of growth mechanisms during the crystal genesis and their morphogenetical influence [1].

However, this nature of non-homogeneous "with history" systems causes limitations to the crystal growth in gel theory. There is no theory of growth from solutions that includes the system evolution in the kinetic formulations of the nucleation and growth processes. Moreover, theory usually deals with homogeneous media in which the concentration becomes only disturbed near the crystal interface, in the so-called "concentration boundary layer" $[2,3]$.

In the case of a " $U$ " tube arrangement [4], where two counter-diffusing reagents meet to generate a sparingly soluble reaction product, the starting boundary conditions are the initial concentrations of the mother solutions and the gel $\mathrm{pH}$. As diffusion time passes by, the concentration of the mother solutions decreases and the gel column becomes non-homogeneous for concentration and $\mathrm{pH}$. Hence, the knowledge of the system history involves finding the concentration and $\mathrm{pH}$ profiles, throughout the gel column, for successive diffusion times. The experimental testing of both, mass-transfer and $\mathrm{pH}$ evolution, is the most rigorous way for such an object, as Prieto et al. [5] have already pointed out.

From these profiles, the supersaturation values may be calculated. However, a rigorous thermodynamic characterization of the system requires knowledge of the reagents activities. Therefore, activity coefficients and concentration of free ions must be found. In this way the Debye-Hückel theory for moderately concentrated solutions may be used [57].

The concentration, $\mathrm{pH}$, and supersaturation profiles form a "snapshot" that shows the spatial distribution of physico-chemical conditions at a 



Fig. 1. Supersaturation evolution. Mother solutions: $0.5 \mathrm{M} \mathrm{SrCl}_{2}$ and $0.5 \mathrm{~N} \mathrm{Na}_{2} \mathrm{CO}_{3}$. (a) Fitting functions $\beta(t)$; (b) supersaturation isolines in the space $x-t$.

particular time. So, the system "history" may be found by means of a sequence of such snapshots taken at known intervals of time. Both spatial and evolutionary aspects are inseparable to explain nucleation and growth phenomena in systems of this kind. Profiles corresponding to the nucleation 
time allow one to interpret the first precipitate location in the diffusion column [8]. However, this instantaneous observation is not enough to justify other aspects of this phenomenon, which are related to the evolutionary history of the zone where the nucleation begins.

This paper deals with aspects of the nucleation behaviour in gels that require the system history to be explained. Since in the start the reagent concentration throughout the gel column is always zero, the physico-chemical evolution of the system may be merely modified by using mother solutions with different initial concentrations. So, it is possible to check the influence of the system history on nucleation phenomena. The study is applied to growth of barium and strontium carbonates in a U-tube gel system. Some conclusions may be however generalized for other substances.

The physico-chemical evolution of the system was studied according to the experimental path of Prieto et al. [5]. In fact, this paper is a close following of an article recently published in this Journal [8], and consequently the experimental details, that are identical to the previous paper, may be consulted there.

\section{The supersaturation rate as kinetic parameter in diffusing-reacting systems}

The "historical" character of diffusing-reacting systems involves finding parameters that reflect the evolution of growth conditions with diffusion time, or what amounts to the same thing, the rate at which the system is moving away from equilibrium everywhere in the diffusion column.

Supersaturation $(\beta)$ is the parameter that reflects the removal from equilibrium of a system. Accordingly, the first stage to study the evolution of growth conditions is to calculate the supersaturation throughout the gel column for different diffusion times. The procedure of calculation, based on the Debye-Hückel theory for moderately concentrated solutions, has been extensively described in a previous paper [8], so it is not considered here.

Supersaturation value at a particular point of the gel column changes continuously with the
Table 1

Supersaturation evolution throughout the gel column; mother solutions: $0.5 \mathrm{M} \mathrm{SrCl}_{2}$ and $0.5 \mathrm{~N} \mathrm{Na}_{2} \mathrm{CO}_{3}$ (strontianite)

\begin{tabular}{|c|c|c|c|c|c|}
\hline \multirow[t]{2}{*}{ Slices } & \multicolumn{5}{|c|}{ Diffusion time (h) } \\
\hline & 750 & 850 & 950 & 1050 & 1150 \\
\hline 1 & - & 1 & 1 & 2 & 3 \\
\hline 2 & - & 1 & 2 & 3 & 6 \\
\hline 3 & - & 1 & 3 & 6 & 11 \\
\hline 4 & - & 1 & 4 & 9 & 16 \\
\hline 5 & - & 2 & 5 & 13 & 25 \\
\hline 6 & - & 2 & 12 & 34 & 66 \\
\hline 7 & - & 3 & 15 & 59 & 148 \\
\hline 8 & 1 & 3 & 26 & 110 & 277 \\
\hline 9 & 1 & 4 & 56 & 199 & 468 \\
\hline 10 & 2 & 10 & 105 & 326 & 716 \\
\hline 11 & 3 & 22 & 255 & 668 & 1269 \\
\hline 12 & 6 & 54 & 478 & 1047 & 1821 \\
\hline 13 & 19 & 204 & 846 & 1590 & 2542 \\
\hline 14 & 31 & 313 & 1330 & 2300 & 3461 \\
\hline 15 & 114 & 754 & 2007 & 3152 & 4440 \\
\hline 16 & 220 & 1391 & 2790 & 4068 & 5413 \\
\hline 17 & 573 & 1709 & 3367 & 4776 & 6196 \\
\hline 18 & 791 & 2041 & 3830 & 5312 & 6760 \\
\hline 19 & 1131 & 2377 & 4087 & 5592 & 7067 \\
\hline 20 & 1456 & 2711 & 4338 & 6002 & 7622 \\
\hline 21 & 1858 & 3032 & 4488 & 6020 & 7543 \\
\hline 22 & 2227 & 3330 & 4612 & 5998 & 7410 \\
\hline 23 & 2513 & 3593 & 4715 & 5967 & 7275 \\
\hline 24 & 2750 & 3808 & 4799 & 5960 & 7194 \\
\hline 25 & 2902 & 3961 & 4868 & 6001 & 7206 \\
\hline 26 & 2905 & 4038 & 4961 & 6150 & 7381 \\
\hline 27 & 2815 & 4023 & 5041 & 6413 & 7766 \\
\hline 28 & 2580 & 3899 & 5135 & 6837 & 8420 \\
\hline
\end{tabular}

diffusion time. Consequently, one can find a fitting function $\beta(t)$ that reflects the sequence of experimental values of supersaturation.

Fig. 1a shows such an adjustement for diffusion from $0.5 \mathrm{M} \mathrm{SrCl}_{2}$ and $0.5 \mathrm{~N} \mathrm{Na} \mathrm{CO}_{3}$ mother solutions. The corresponding experimental data are listed in table 1 . Values of supersaturation-time have been fitted to third degree polynomia by the least squares method. Correlation coefficients were greater than 0.999 in all the cases. Fig. 1b shows a map of supersaturation isolines in the space $x-t$. From this map, supersaturation profiles throughout the gel column may be figured out. Complete mass-transfer and supersaturation data used for this work are available to readers. An exhaustive report is, however, beyond the scope of the present work. 

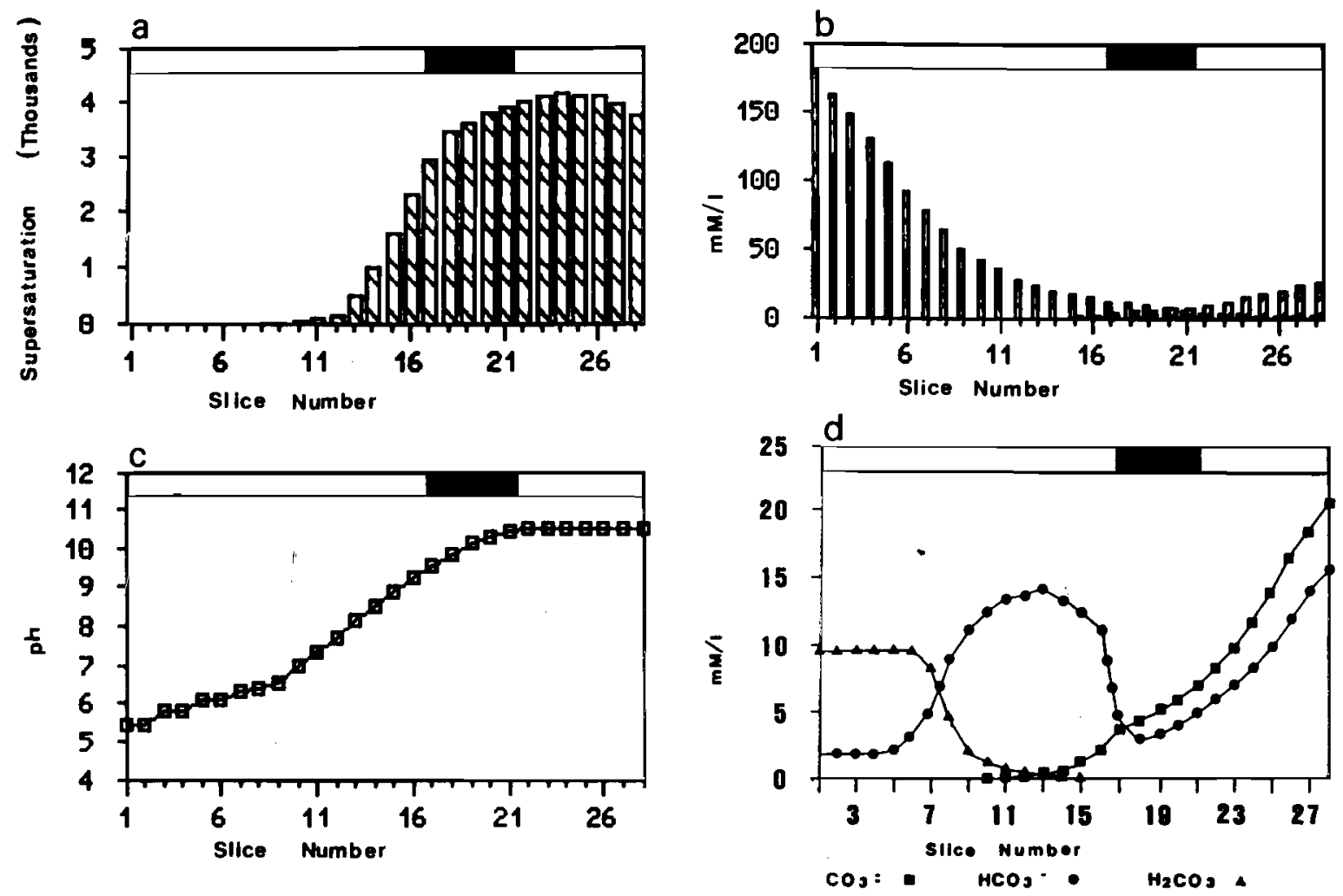

Fig. 2. Profiles at the nucleation time. Mother solutions: $0.5 \mathrm{M} \mathrm{SrCl}_{2}$ and $0.5 \mathrm{~N} \mathrm{Na}{ }_{2} \mathrm{CO}_{3}$. The reaction zone is outlined in the upper part of the graphics. (a) Supersaturation; (b) concentration of $\mathrm{Sr}^{2+}$ and $\mathrm{CO}_{3}^{2-}$; (c) pH; (d) distribution of carbonatic species along the gel column.

The $\beta(t)$ functions allow one to define an evolutionary parameter, the supersaturation rate, that states the speed at which the system is moving away from equilibrium. The supersaturation rate is given by:

$R_{\beta}=\partial \beta / \partial t$.

Therefore, the dimensional formula of supersaturation rate is given by $\left[T^{-1}\right]$ and one can use $\mathrm{h}^{-1}$ as a unit. Such a concept has been mentioned in different works of crystal growth in gels [1,9], but it has never been formalized to account for experiments.

Values of $R_{\beta}$, at a particular time and position, may be obtained from the derivative of corresponding $\beta(t)$ functions. Table 2 shows the supersaturation rate values, at the nucleation time, for different starting conditions. Both sustances, strontianite and witherite, are considered.
In addition to concentration, $\mathrm{pH}$ and supersaturation, the supersaturation rate along the gel column is non-homogeneous. Figs. 3 and 4 deal with supersaturation rate profiles, at the nucleation time, for different initial concentrations of mother solutions. It is worth noting that supersaturation profiles show a maximum, whose location will be discussed later.

\section{Nucleation behaviour: spatial aspects}

The most obvious spatial phenomenon of nucleation in systems of this kind is the location of precipitates in particular places of the diffusion column. In a previous paper [8], the authors correlate concentration, $\mathrm{pH}$ and supersaturation profiles, corresponding to nucleation time, with the first precipitate positions. The findings confirm 



Fig. 3. Supersaturation rate profiles at the nucleation time (strontianite). Mother solutions: (a) $0.5 \mathrm{M} \mathrm{SrCl}{ }_{2}$ and $0.5 \mathrm{~N} \mathrm{Na}_{2} \mathrm{CO}_{3}$; (b) $0.5 \mathrm{M} \mathrm{SrCl}_{2}$ and $0.1 \mathrm{~N} \mathrm{Na}_{2} \mathrm{CO}_{3} ;$ (c) $0.3 \mathrm{M} \mathrm{SrCl}_{2}$ and $0.3 \mathrm{~N} \mathrm{Na}_{2} \mathrm{CO}_{3} ;$ (d) $0.1 \mathrm{M} \mathrm{SrCl}$ and $0.1 \mathrm{~N} \mathrm{Na}_{2} \mathrm{CO}_{3}$.

that supersaturation profiles are not enough to explain nuclei locations. Following Henisch and García-Ruiz [10], the reagent concentration ratio is not irrelevant for a stochastically controlled system of limited particle mobility, like nucleation in a gel. In a gel, one is dealing with time-dependent macroscopic concentration gradients in which the reagent concentration ratio takes every kind of value along the diffusion column. Thus, as a rule for weakly soluble sustances, first precipitate locations do not agree with maxima of supersaturation profiles. On the contrary, nucleation occurs in regions where the ratio of reagents concentration (stated in equivalents) is near to unit.

This behaviour is specially evident in the case of strontianite, witherite and other sustances with low solubility. Fig. 2 shows profiles of different physico-chemical variables at the nucleation time (912 h) for $0.5 \mathrm{M} \mathrm{SrCl}_{2}$ and $0.5 \mathrm{~N} \mathrm{Na}_{2} \mathrm{CO}_{3}$ mother solutions. One may note that the supersaturation maximum is shifted towards the $\mathrm{CO}_{3}^{2-}$ reservoir (slices 23-26). However, the nuclei fill up a region (slices 17-21) where the $\left[\mathrm{Sr}^{2+}\right] /\left[\mathrm{CO}_{3}^{2-}\right]$ ratio takes values between 1.98 and 0.77 (fig. 2b). Analogous occurrences may be observed for the other initial concentrations of mother solutions and in the case of witherite growth.

Supersaturation maximum location is related to the distribution of "carbon-containing" species which is in turn $\mathrm{pH}$-dependent. So, at a $\mathrm{pH}=10.5$, the contribution of $\mathrm{HCO}_{3}^{-}$and $\mathrm{H}_{2} \mathrm{CO}_{3}^{0}$ to the total carbon concentration is low compared to that of $\mathrm{CO}_{3}^{2-}$. On the contrary, at lower $\mathrm{pH}$, $\mathrm{HCO}_{3}^{-}$and $\mathrm{H}_{2} \mathrm{CO}_{3}^{0}$ are predominant. On the other hand, fig. $2 \mathrm{c}$ shows that $\mathrm{pH}$ increases from 5.5 in slice 3 to 10.5 in slice 20, and remains constant beyond this slice. Consequently, in the low $\mathrm{pH}$ region, $\mathrm{H}_{2} \mathrm{CO}_{3}^{0}$ is the prevailing species (slices 

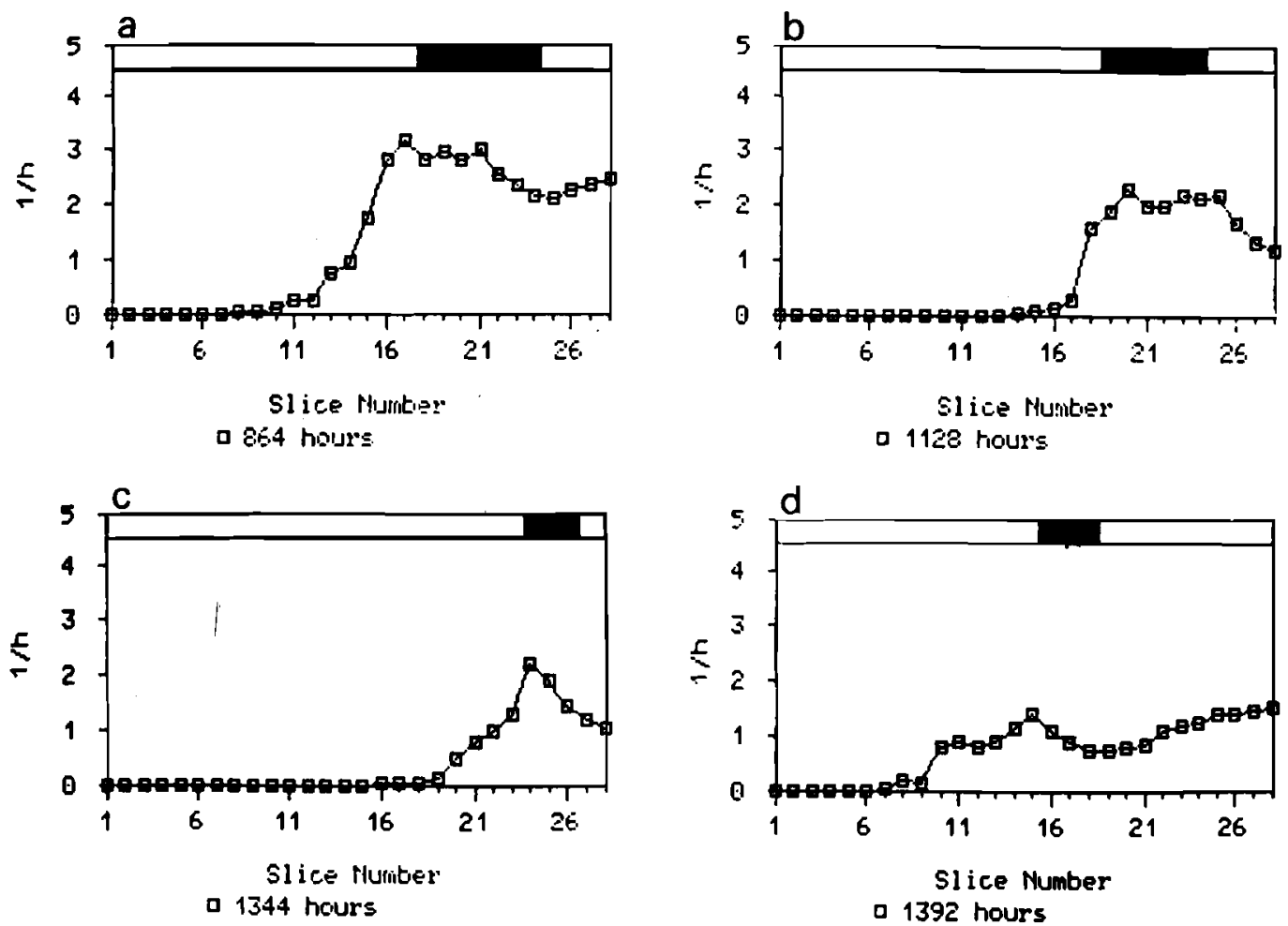

Fig. 4. Supersaturation rate profiles at the nucleation time (witherite). Mother solutions: (a) $0.5 \mathrm{M} \mathrm{BaCl}_{2}$ and $0.5 \mathrm{~N} \mathrm{Na}{ }_{2} \mathrm{CO}_{3}$; (b) $0.5 \mathrm{M}$ $\mathrm{BaCl}_{2}$ and $0.3 \mathrm{~N} \mathrm{Na}_{2} \mathrm{CO}_{3}$; (c) $0.5 \mathrm{M} \mathrm{BaCl}_{2}$ and $0.1 \mathrm{~N} \mathrm{Na}_{2} \mathrm{CO}_{3} ;$ (d) $0.1 \mathrm{M} \mathrm{BaCl}_{2}$ and $0.1 \mathrm{~N} \mathrm{Na} \mathrm{CO}_{3}$.

1-7), $\mathrm{HCO}_{3}^{-}$predominates in slices 7 to 16 , and finally, $\mathrm{CO}_{3}^{2-}$ ions are the most abundant in slices 16 to 20 (fig. 2d).

On the basis of the above, the $\mathrm{CO}_{3}^{2-}$ concentration is only important in the high $\mathrm{pH}$ region, near the $\mathrm{Na}_{2} \mathrm{CO}_{3}$ reservoir. This explains that maximum values of supersaturation are constrained to this region of the system.

The sloping segment of the $\mathrm{pH}$ profile is due to the high sensibility of $\mathrm{pH}$ with respect to the total concentration of carbonates: $\mathrm{pH}$ changes from 5.5 for $[\mathrm{C}]_{\text {total }} \leq 14 \mathrm{mM} / 1$ to 10.5 for $[\mathrm{C}]_{\text {total }} \geq$ $35 \mathrm{mM} / 1$. Further concentration increases do not result in meaningful $\mathrm{pH}$ changes.

In the region where $\mathrm{pH}$ increases the high $\mathrm{CO}_{3}^{2-}$ concentration gradient is a result of two cooperating effects, the whole concentration gradient of carbonatic species and the $\mathrm{pH}$ gradient. It is in this place where the $\mathrm{CO}_{3}^{2-}$ concentration becomes equal to the $\mathrm{Sr}^{2+}$ one and nucleation occurs. The equality range condition is more restrictive in the case of strontianite because of the higher solubility of witherite. However, in both cases the nucleation probability tends to zero rather rapidly as $\left[\mathrm{Sr}^{2+}\right] /\left[\mathrm{CO}_{3}^{2-}\right]$ or $\left[\mathrm{Ba}^{2+}\right] /\left[\mathrm{CO}_{3}^{2-}\right]$ depart significantly from unit.

There is no correspondence between supersaturation and supersaturation rate profiles. At a particular time, supersaturation and supersaturation rate maxima do not necessarily coincide. On the contrary, supersaturation rate maxima coincide with the sloping segment of $\mathrm{pH}$ and hence with the reaction zone. Fig. 3 shows the $R_{\beta}$ profiles at the nucleation time for different initial concentrations. Fig. 4 shows the same kind of profiles for witherite growth. First, precipitate locations are outlined in the upper part of the graphics. The results are summarized in table 3, in which are also collected the $\mathrm{pH}$ values corresponding to the limits of the reaction zones. The agreement of these zones with the upward segment of $\mathrm{pH}$ becomes evident. 
Correspondence between nucleation zones and $R_{\beta}$ maxima is a factor of great kinetic importance. The high values of $R_{\beta}$ in this place guarantee maintenance of supersaturation level during and after the nucleation process.

\section{Nucleation behaviour: evolutionary aspects}

Critical supersaturation is a classical concept of the nucleation theory with great kinetic meaning. The theory of homogeneous nucleation from solution suggests that the nucleation rate is dependent

Table 2

Supersaturation rate $\left(\mathrm{h}^{-1}\right)$, at the nucleation time, for different start conditions (strontianite)

\begin{tabular}{cclll}
\hline Slices & \multicolumn{5}{l}{ Mother solutions: $\mathrm{SrCl}_{2}$ and $\mathrm{Na}_{2} \mathrm{CO}_{3}$} \\
\cline { 2 - 5 } & $912 \mathrm{~h}$ & $1250 \mathrm{~h}$ & $1344 \mathrm{~h}$ & $1632 \mathrm{~h}$ \\
& $0.5 \mathrm{M}-0.5 \mathrm{~N}$ & $0.3 \mathrm{M}-0.3 \mathrm{~N}$ & $0.5 \mathrm{M}-0.1 \mathrm{~N}$ & $0.1 \mathrm{M}-0.1 \mathrm{~N}$ \\
\hline 1 & - & - & - & - \\
2 & 0.01 & - & - & - \\
3 & 0.02 & - & - & - \\
4 & 0.03 & - & - & - \\
5 & 0.05 & - & - & - \\
6 & 0.12 & - & - & - \\
7 & 0.26 & - & - & - \\
8 & 0.48 & 0.01 & - & - \\
9 & 0.83 & 0.01 & - & - \\
10 & 1.29 & 0.01 & 0.01 & 0.01 \\
11 & 2.36 & 0.02 & 0.01 & 0.01 \\
12 & 4.04 & 0.04 & 0.01 & 0.01 \\
13 & 5.66 & 0.07 & 0.02 & 0.09 \\
14 & 7.88 & 0.13 & 0.04 & 0.20 \\
15 & 9.82 & 0.33 & 0.07 & 0.32 \\
16 & 11.63 & 1.09 & 0.12 & 0.47 \\
17 & 13.22 & 1.75 & 0.19 & 0.63 \\
18 & 14.23 & 2.26 & 0.50 & 0.80 \\
19 & 16.91 & 3.08 & 1.12 & 0.99 \\
20 & 16.08 & 2.46 & 1.57 & 1.19 \\
21 & 14.80 & 2.36 & 2.15 & 1.44 \\
22 & 13.40 & 2.25 & 2.76 & 1.70 \\
23 & 12.16 & 2.18 & 4.11 & 1.95 \\
24 & 11.35 & 2.19 & 5.02 & 1.88 \\
25 & 11.31 & 2.24 & 5.13 & 1.80 \\
26 & 12.12 & 2.53 & 4.56 & 1.63 \\
27 & 13.37 & 2.63 & 4.03 & 1.47 \\
28 & 14.26 & 2.33 & 3.31 & 1.35 \\
\hline & & & & \\
\hline
\end{tabular}

on the supersaturation in accordance with an expression of the form:

$J=\Omega^{\prime} \exp \left(-\frac{\delta \sigma^{3} \Omega^{2}}{k^{3} T^{3}(\ln \beta)^{2}}\right)$,

where $\delta$ is a shape factor, $\sigma$ is the surface free energy, and $\Omega$ the molecular volume. The pre-exponential factor $\Omega^{\prime}$ is related to the growth of the critical nucleus to become supercritical, and involves the volume diffusion step.

From this relation it is clear that the nucleation rate is a very sharp function of supersaturation and it is usual to define the critical supersaturation as corresponding to the rate of 1 nucleus $/ \mathrm{s}$. $\mathrm{cm}^{3}$ [11]. The critical supersaturation corresponds then to the breakdown of the metastable state.

In time-dependent and non-homogeneous systems, the critical supersaturation concept, however, becomes ambiguous. In the present case, experiments demonstrate that the starting boundary conditions determine the supersaturation level when nucleation begins. Consequently, we then use the term "supersaturation threshold" $\beta$ * for this level, on the understanding that critical supersaturation is a different concept.

For similar reasons, the induction period concept cannot be applied to systems of this kind. This is one of the parameters which traditionally has been used to describe nucleation kinetics from solution. It is defined as the time elapsed between the creation of the supersaturated state and the appearance of a critical nucleus [11]. As there is no method of detecting critical nuclei, their formation has to be inferred from changes in some physical property of the solution. The time measured in this way is called the "experimental induction period for crystallization", and is obviously greater than the actual induction time. However, we cannot use the induction period concept in a medium where composition and supersaturation change markedly in space and time. So the term experimental induction time, used in this work, is a more complex term which depends on the supersaturation evolution in the system.

As mentioned earlier, the physico-chemical evolution of the system may be modified by using mother solutions with different initial concentra- 
Table 3

Spatial aspects of the nucleation behaviour

\begin{tabular}{lllllll}
\hline $\begin{array}{l}\text { Mother solution } \\
\mathrm{Sr}-\mathrm{Bat}\end{array}$ & $\mathrm{Na}_{2} \mathrm{CO}_{3}$ & $\begin{array}{l}\text { Precipitate } \\
\text { location } \\
\text { (slices) }\end{array}$ & $\begin{array}{l}\mathrm{M}^{2+} / \mathrm{CO}_{3}^{2-} \\
\text { (range) }\end{array}$ & $\begin{array}{l}\mathrm{pH} \\
\text { (range) }\end{array}$ & $\begin{array}{l}\beta, \\
\text { maximum } \\
\text { location }\end{array}$ & $\begin{array}{l}R_{\beta}, \\
\text { maximum } \\
\text { location }\end{array}$ \\
\hline Strontianite & & & & & & \\
$0.5 \mathrm{M}$ & $0.5 \mathrm{~N}$ & $17-21$ & $1.98-0.77$ & $9.5-10.5$ & $24-25$ & 19 \\
$0.5 \mathrm{M}$ & $0.3 \mathrm{~N}$ & $20-23$ & $1.59-0.88$ & $9.5-10.5$ & 24 & 22 \\
$0.3 \mathrm{M}$ & $0.5 \mathrm{~N}$ & $16-18$ & $1.80-0.86$ & $9.5-10$ & 17 & 16 \\
$0.3 \mathrm{M}$ & $0.3 \mathrm{~N}$ & $18-20$ & $1.73-0.91$ & $9-10$ & $23-24$ & 19 \\
$0.5 \mathrm{M}$ & $0.1 \mathrm{~N}$ & $23-27$ & $2.02-0.63$ & $8.5-10$ & $27-28$ & $24-25$ \\
$0.1 \mathrm{M}$ & $0.5 \mathrm{~N}$ & $14-17$ & $1.59-0.85$ & $9.5-10$ & 22 & 16 \\
$0.3 \mathrm{M}$ & $0.1 \mathrm{~N}$ & $22-24$ & $1.96-0.74$ & $9-9.5$ & $25-26$ & 23 \\
$0.1 \mathrm{M}$ & $0.3 \mathrm{~N}$ & $17-19$ & $1.58-0.73$ & $8.5-10$ & 23 & $18-19,23$ \\
$0.1 \mathrm{M}$ & $0.1 \mathrm{~N}$ & $19-23$ & $1.90-0.72$ & $8.5-9.5$ & 28 & 23 \\
Witherite & & & & & & $17-21$ \\
$0.5 \mathrm{M}$ & $0.5 \mathrm{~N}$ & $18-22$ & $3.30-0.50$ & $10-10.5$ & $26-27$ & 16 \\
$0.3 \mathrm{M}$ & $0.5 \mathrm{~N}$ & $16-19$ & $2.06-0.78$ & $9.5-10.5$ & 28 & $20-23$ \\
$0.5 \mathrm{M}$ & $0.3 \mathrm{~N}$ & $19-24$ & $3.50-0.50$ & $9.5-10.5$ & $27-28$ & 19 \\
$0.3 \mathrm{M}$ & $0.3 \mathrm{~N}$ & $17-20$ & $3.58-0.69$ & $8.5-10$ & 24 & 24 \\
$0.5 \mathrm{M}$ & $0.1 \mathrm{~N}$ & $24-26$ & $4.70-0.80$ & $10-10.5$ & $27-28$ & 15 \\
$0.1 \mathrm{M}$ & $0.5 \mathrm{~N}$ & $16-18$ & $2.30-0.70$ & $9.5-10.5$ & 28 & $20-23$ \\
$0.3 \mathrm{M}$ & $0.1 \mathrm{~N}$ & $20-22$ & $5.32-0.66$ & $8.5-9.5$ & 28 & $18-21$ \\
$0.1 \mathrm{M}$ & $0.3 \mathrm{~N}$ & $18-20$ & $1.12-0.79$ & $9.5-10$ & 25 & $21-24$ \\
$0.1 \mathrm{M}$ & $0.1 \mathrm{~N}$ & $19-21$ & $7.52-0.91$ & 10.5 & 28 & \\
\hline
\end{tabular}

tions. That makes possible to compare analogous systems with different histories. Table 4 collects data which are in relation with the system evolution. Values of $\beta^{*}$ and $R_{\beta}$ are mean values corresponding to the crystallization zone. The data are ordered according to the experimental induction time.

The first outcome is the correspondence between initial concentrations and induction periods. As solution reservoir concentrations are increased, the induction period decreases. That is to be expected because increasing initial concentrations means increasing the whole supersaturation rate of the system. Local values of $R_{\beta}$ at the crystallization zone reflect this statement, as table 4 shows.

The relation between supersaturation threshold and supersaturation rate, however, is not so evident. The results show that the metastability level goes with high supersaturation rate. Hence, the supersaturation threshold is a variable that depends on the system evolution, changing within wide margins (3742 to 457 for the experiments
Table 4

Evolutionary aspects of the nucleation behaviour

\begin{tabular}{lllll}
\hline Mother solutions & $\begin{array}{l}\text { Induction } \\
\text { time } \\
\text { (h) }\end{array}$ & $\beta^{*}$ & $R_{\beta}$ & $\begin{array}{l}\text { Number } \\
\text { of }\end{array}$ \\
& $\mathrm{Na}_{2} \mathrm{CO}_{3}$ & & nuclei \\
\hline
\end{tabular}

Strontianite

$\begin{array}{lrrrrr}0.5 \mathrm{M} & 0.5 \mathrm{~N} & 912 & 3742 & 15.11 & 14 \\ 0.5 \mathrm{M} & 0.3 \mathrm{~N} & 1050 & 3234 & 7.46 & 12 \\ 0.3 \mathrm{M} & 0.5 \mathrm{~N} & 1080 & 1893 & 4.68 & 10 \\ 0.3 \mathrm{M} & 0.3 \mathrm{~N} & 1200 & 1269 & 3.00 & 6 \\ 0.5 \mathrm{M} & 0.1 \mathrm{~N} & 1344 & 1390 & 4.53 & 5 \\ 0.1 \mathrm{M} & 0.5 \mathrm{~N} & 1315 & 1285 & 3.35 & 7 \\ 0.3 \mathrm{M} & 0.1 \mathrm{~N} & 1440 & 946 & 2.99 & 3 \\ 0.1 \mathrm{M} & 0.3 \mathrm{~N} & 1580 & 663 & 2.60 & 3 \\ 0.1 \mathrm{M} & 0.1 \mathrm{~N} & 1632 & 457 & 1.45 & 2\end{array}$

Witherite

\begin{tabular}{lrrrrr}
$0.5 \mathrm{M}$ & $0.5 \mathrm{~N}$ & 864 & 889 & 2.60 & 4 \\
$0.3 \mathrm{M}$ & $0.5 \mathrm{~N}$ & 1080 & 435 & 1.14 & 4 \\
$0.5 \mathrm{M}$ & $0.3 \mathrm{~N}$ & 1128 & 865 & 2.05 & 4 \\
$0.3 \mathrm{M}$ & $0.3 \mathrm{~N}$ & 1250 & 354 & 0.72 & 4 \\
$0.5 \mathrm{M}$ & $0.1 \mathrm{~N}$ & 1344 & 470 & 1.50 & 2 \\
$0.1 \mathrm{M}$ & $0.5 \mathrm{~N}$ & 1392 & 434 & 0.80 & 3 \\
$0.3 \mathrm{M}$ & $0.1 \mathrm{~N}$ & 1464 & 138 & 0.50 & 2 \\
$0.1 \mathrm{M}$ & $0.3 \mathrm{~N}$ & 1536 & 283 & 0.57 & 2 \\
$0.1 \mathrm{M}$ & $0.1 \mathrm{~N}$ & 1704 & 82 & 0.40 & 2 \\
\hline
\end{tabular}


collected here). At any rate, the supersaturation level is rather high in all cases, which is expected for media with limited particle mobility and for weakly soluble substances. Both cooperating effects help the metastability and consequently increase the supersaturation threshold. The lower $\beta^{*}$ values for witherite are again explained because of its higher solubility.

Table 4 also collects the total number of nuclei that appear (under magnification $\times 500$ ) $24 \mathrm{~h}$ after nucleation time. This number decreases with $\beta^{*}$, as expected from classical nucleation assessments.

\section{Conclusions}

Nucleation behaviour in diffusing-reacting systems requires spatial considerations to explain the observed phenomena. First, precipitate locations may be justified from the concentration, $\mathrm{pH}$ and supersaturation profiles at the nucleation time. Experimental results demonstrate that the critical supersaturation condition is insufficient to account for nucleation in a double-diffusive system. A second condition, the "equality range" of reagent concentrations, must be also fulfilled.

The place where nucleation begins coincides with the sloping segment of $\mathrm{pH}$. In this region the high $\mathrm{CO}_{3}^{2-}$ concentration gradient is a result of two cooperating effects, the whole concentration gradient of carbonatic species and the $\mathrm{pH}$ gradient. It is in this place where the $\mathrm{CO}_{3}^{2-}$ concentration quickly equalizes to the cation concentration and nucleation occurs. The coincidence of this zone with the supersaturation rate maximum is also in relation with the fast rise of the $\mathrm{CO}_{3}^{2-}$ concentration. Moreover, correspondence between nucleation zone and $R_{\beta}$ maximum guarantees maintenance of supersaturation level during the nucleation process. Thus, besides supersaturation, equality range and supersaturation rate cooperate kinetically to produce nucleation at a specific place of the column.

However, these spatial considerations are not enough to explain other aspects of nucleation where the evolutionary history of the system be- comes an important factor. The supersaturation rate has an important bearing on the supersaturation level at the nucleation time and on the nucleation density. The large variation of:nuclea- tion threshold $\beta^{*}$ with $R_{\beta}$ has no simple explanation. Induction time is characteristic for each supersaturation and for each reactant concentration ratio, and both parameters continuousty change in the nucleation zone. Computer modelization of nucleation for different supersaturation rates may, make clear this phenomenon. Future papers will deal with this matter.

\section{Acknowledgments}

This work was done as a part of project No. 472/84 supported by CAICYT (Ministry of Education and Science of Spain). The authors are indebted to the Laboratorio de Mineralogía y Edafologia de la Universidad de Castilla-La Mancha, for the $\mathrm{C}-\mathrm{N}-\mathrm{S}$ analysis.

\section{References}

[1] J.M. García-Ruiz, J. Crystal Growth 75 (1986) 441.

[2] F. Rosenberger, Fundamentals of Crystal Growth: Macroscopic Equilibrium and Transport Concepts (Springer, Berlin, 1979).

[3] R.J. van Rosmalen, Crystal Growth Processes: The Role of Steps and of Mass-Transfer in the Fluid Phase, Thesis, Technical University of Delft (1977).

[4] H.K. Henisch, Crystal Growth in Gels (Pennsylvania State University Press, University Park, PA, 1970).

[5] M. Prieto, C. Viedma, V. Lopez-Acevedo, J.L. MartinVivaldi and S. López-Andrés, J. Crystal Growth 92 (1988) 61.

[6] H.E. Lundager Madsen, Nephrologie 5 (1984) 151.

[7] L. Amathieu and R. Boistelle, J. Crystal Growth 88 (1988) 183.

[8] M. Prieto, L. Fernández-Diaz and S. López-Andrés, J. Crystal Growth 98 (1989) 447.

[9] H.K. Henisch and J.M. Garcia-Ruiz, J. Crystal Growth 75 (1986) 195.

[10] H.K. Henisch and J.M. Garcia-Ruiz, J. Crystal Growth 75 (1986) 203.

[11] A.G. Walton, in: Nucleation, Ed. A.C. Zettlemoyer (Dekker, New York, 1969). 